\title{
New Trends in Multiscale and Multiphysics Simulation of Transport Phenomena in Novel Engineering Systems
}

\author{
D. Lakehal ${ }^{\dagger}$ \\ ASCOMP GmbH, Technoparkstrasse 1, Zurich, 8005, Switzerland \\ $\dagger$ Corresponding Author Email: lakehal@ascomp.ch
}

(Received July 16, 2010; accepted March 13, 2011)

\begin{abstract}
The paper reports on the progress made in predicting large- and small-scale single and two-phase flows with heat transfer using the CMFD code TransAT. In the multi-phase context, the code uses the Level Set approach as the "Interface Tracking Method" of reference. The solver incorporates phase-change capabilities, surface tension and triple-line dynamics models, Marangoni effects, electric and magnetic fields, and a wall micro-film sub-grid scale model for lubrication. Complex 3D examples shown here were treated using a fully automatized version of the code, using the Immersed Surfaces Technique (IST) to map complex components into a simple rectangular Cartesian grid. It is shown that real coupled two-phase heat transfer (conjugate) problems are within reach of modern CMFD code using interface tracking, with relatively fast response times: 3D coupled two-phase flow heat transfer can run on a simple Linux PC cluster within $24 \mathrm{H}$ time.
\end{abstract}

Keywords: Multi-phase, Multi-physics, TransAT, CMFD.

\section{INTRODUCTION}

Computational fluid dynamics (CFD) and its multi-fluid variant CMFD are playing a major role in the design of novel energy and process engineering related systems. These flows are in general multi-faceted in terms of physics, appearing sometimes under as multi-phase or components, sometimes featuring conjugate heat transfer, or chemical reaction. Convective-conductive heat transfer within multi-phase flow is a combined transport phenomenon that exists in a multitude of thermal-flow systems, including thermal management in electronics boards (PCB), heat exchangers, heat pipes and micro-channels. The issue here is to arrive at a CFD or CMFD tool capable to deal with the various facets at once; a sort of multi-scale, multi-physics computational strategy. Microfluidics flows for instance represent a perfect class of examples: the flows are associated with free-surface motion evolving as falling films, spreading and de-wetting of (complex) liquids on solid or liquid substrates, chemical reaction of binary mixtures, micro-bubbles and droplet motion, phase change or transition. The control of such micro-flow systems (using pressure, Marangoni, electro-wetting, Ferro-fluidics, and acoustics) is central to future technological advances in emerging technologies, e.g. lab-on-the-chip and microreactor technology.

We shall discuss various multi-scale, multi-physics examples computed using the CMFD code TransAT. The first part introduces the modeling and algorithmic approaches and their applicability to various single and multiphase flow problems, with variable degree of complexity; conjugate heat transfer, icro-scale, free surfaces, etc. Besides the complexity of the physics, we introduce the Immersed Surfaces Technique (IST) to mesh complex geometries, which helps save time in grid generation while reducing numerical diffusion.

\section{INTERFACIAL TWO-PHASE FLOW}

Free-surface and interfacial flows refer to two-phase flow problems that involve two or more immiscible fluids separated by sharp interfaces which evolve in time. Typically, when the fluid on one side of the interface is a gas that exerts shear (tangential) stress upon the interface, the latter is referred to as a free surface. Interface tracking schemes are methods that are able to locate the interface, not by following the interface in a Lagrangian sense (by following marker points that reside on the interface), but by capturing the interface by keeping track, in an Eulerian sense (the grid is fixed), of the evolution of an appropriate field such as a level-set function, a volume-fraction field, a phase-indicator field. Examples include the Level-Set method (Sussman et al. 1994), in which the interface is considered to be a level surface of a function that is defined over all space, and the Volume-Of-Fluid method (Hirt \& Nichols 1981), in which the location of the interface is captured by keeping track of the volume fraction of each computational cell in the grid with respect to one of the fluid phases: cells that have a volume fraction of zero or unity do not contain an interface, whereas those that have a fractional value do. 
Be it as it may, the literature has made it such that these methods are all known as Interface Tracking Methods (ITM); see Lakehal et al. (2002).

\subsection{Interface Tracking Methods}

ITM methods are used for the prediction of two-phase flows requiring precise interface identification, such as breakup of large bubbles, droplets or liquid jets. The key to the methods is the use of a single-fluid set of conservation equations with variable material properties and surface forces, i.e. the single-fluid formalism. The concept is attractive, since it offers the prospect of a more accurate strategy than that offered by the two-fluid formalism, while minimizing modeling assumptions. The fluid motion and heat transfer equations under incompressible flow conditions expressed within the single-fluid formalism (or ITM) take the following form:

$$
\begin{aligned}
& \nabla \cdot \mathbf{u}=0 \\
& D_{t}(\rho \mathbf{u})=-\nabla p+\nabla \cdot \mu(\nabla \mathbf{u})+F_{s}(\phi)+F_{c}(\phi) \\
& D_{t}(\rho C p T)=\nabla \cdot \lambda(\nabla T)+Q^{\prime \prime}
\end{aligned}
$$

where $\rho$ is the density, $p$ is the pressure, and $\mu$ is the viscosity. The last RHS terms in (2) represent the surface tension force (Fs) and its contact-line wall contribution $(\mathrm{Fc})$, with $\mathrm{n}$ standing for the normal vector to the interface, $\kappa$ for the surface curvature, $\gamma$ for the surface tension coefficient of the fluid, and $\delta$ for a smoothed Dirac delta function centered at the interface. In Eq. (3), $\mathrm{T}$ is the temperature, $\mathrm{Cp}$ is the heat capacity of the fluids, and $\lambda$ is the heat conductivity.

In the Level Set technique, the interface between immiscible fluids is represented by a continuous function $(\phi)$, representing the distance to the interface that is set to zero on the interface, is positive on one side and negative on the other. This way, both fluids are identified, such that the location of the physical interface is associated with the zero level. The material properties, the body and surface forces in Eq. (2) are all locally dependent on function $\phi$, the evolution equation of which in the presence of phase change is

$\partial_{t}(\phi)+\mathbf{u} . \nabla \phi=\dot{m} / \rho|\nabla \phi|$,

where $\dot{m}$ stands for the mass transfer rate, which can be either directly determined using the energy jump across the interface, or modeled using heat transfer correlations (Lakehal et al. 2002). The treatment of wetting dynamics is based on the physical forces associated with triple lines included in the momentum equations as a source term. The contact line force accounts for the interfacial tension between the fluids and the equilibrium contact angle, and is obtained by considerations similar to the derivation of Young's Law, referred to as the unbalanced Young force. In the case of the static contact angle treatment, the wall value of the level set is calculated such that the contact angle is always equal to the equilibrium contact angle specified.

\subsection{TransAT@ CMFD Flow Software}

The CMFD code TransAT $\odot$ of ASCOMP GmbH (2010) is a multi-physics, finite-volume code based on solving multi-fluid Navier-Stokes equations on structured multi-block meshes. MPI parallel based algorithm is used in multi-blocks. Grid arrangement is collocated. The solver is pressure based, corrected using the Karki-Patankar technique for compressible flows. High-order time marching and convection schemes can be employed; up to $3^{\text {rd }}$ order Monotone schemes in space (e.g. Quick scheme bounded using a TVD limiter), and $3^{\text {rd }}$ to $5^{\text {th }}$ order Runge-Kutta scheme in time. The diffusive fluxes are differenced using the 2 nd order central scheme.

Multiphase flows are tackled using Level Sets, VOF and Phase-Field for both laminar and turbulent flows. The solver incorporates phase change, surface tension and triple-line dynamics capabilities, Marangoni effects, and sub-grid scale model for ultra-thin film lubrication.

\subsection{The Immersed Surfaces Technique}

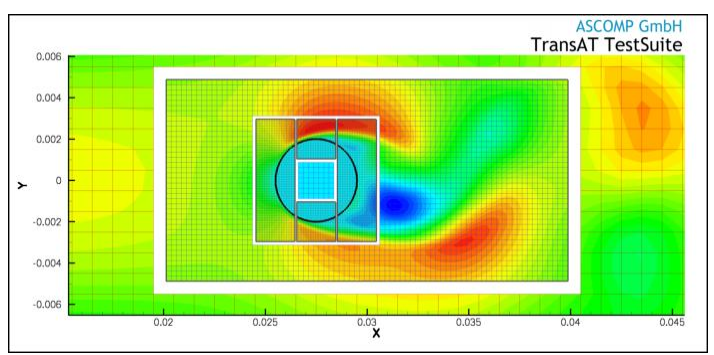

Fig. 1. Flow past a cylinder surface using IST

The Immersed Surfaces Technology (IST) has been developed by ASCOMP GmbH (to the best of our knowledge), although other similar approaches have been developed in parallel. The underpinning idea is inspired from Interface Tracking techniques for two-phase flows (VOF and Level Sets), where free surfaces are described by a convection equation for the phase color function. In IST the solid is described as the second 'phase', with its own thermo-mechanical properties. The technique differs from the Immersed Boundaries methods (Mittal and Iaccarino 2005), in that the jump condition at the solid surface is implicitly accounted for, not via direct momentum forcing (using the penalty approach) on the Eulerian grids. It has the major advantage to solve conjugate heat transfer problems, in that conduction inside the body is directly linked to external fluid convection. The flow past a circular cylinder shown in Fig. 1 is an illustrative example. The solid, which is defined by its external boundaries using the solid level set function denoted by $\phi s$, is first immersed into a Cartesian mesh. Like in fluid-fluid flows, solid level set function is defined as a distance to the surface; is zero at the surface, negative in the fluid and positive in the solid. The treatment of viscous shear at the solid surfaces is handled very much the same way as in all CFD codes, where wall indices are known form a boundary condition files.

\section{Single-PHASe CASE Studies}

\subsection{Cooling of Electronics PCB Boards}

Cooling of electronic boards is typically achieved by means of fan-based convection. The user is interested to evaluate the cooling effectiveness of the design, using fans of various characteristics, placed at various 
locations. CMFD helps provide a faithful picture of what might happen under a specific set-up. Our dedicated variant of the code TransAT has been designed to help re-design engineering operations. Thanks to IST, generating a 3D set-up (grid, domain, fan dimensioning, material properties set-up, boundary conditions) of a complex board with various components is of the order of one hour.
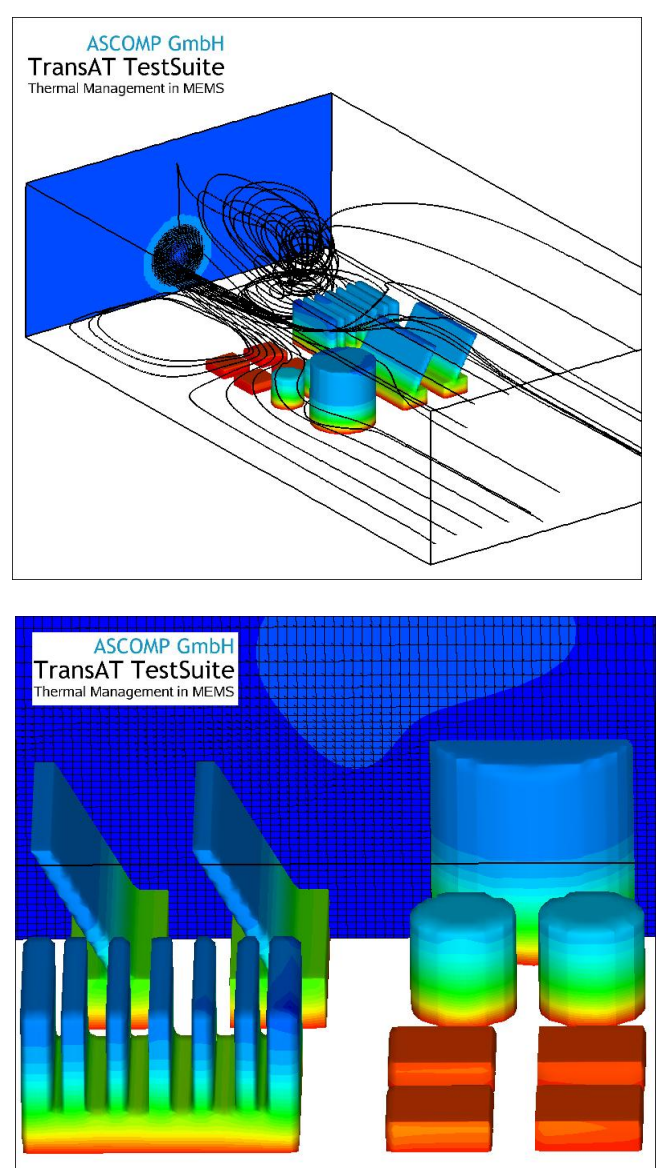

Fig. 2. A typical PCB with various components cooled by a fan. Use is made of IST for meshing.

The flow solution shown in Fig. 2 above reflects the capability of the EST module to map complex-shaped components in a simple Cartesian grid, refined where necessary (around the components). The steady state flow is calculated using the $\mathrm{k}-\varepsilon$ model for turbulence combined with wall functions. The grid consists of $125^{\prime} 000$ cells. Only high order schemes were employed. Figure 2 depicts the effect of fan-generated coolant on the heat transfer from the components to the core flow. In this module the set-up for fan characteristics can be made automatically, specifying for example the number of fans, the hub diameter, the rotational speed and the mean flow of each fan. Thin-sheet elements are artificially thickened as to allow them to be represented by the grid ( 3 cells should cover inner solid areas). Each component can be assigned it mechanical properties and volumetric heat source. New developments in TransAT include treating anisotropic heat transfer on thin-crystal layers, which possess higher conductivity in the vertical direction (to suck heat from the hot spot down to the substrate).

\subsection{Flow in Microrheometric Devices}

Research is nowadays conducted by microfluidics manufacturers to develop a novel, efficient microfluidic "rheometer-on-a-chip" capable of achieving high strain rates, such that it can be used to measure the effective extensional properties of dilute polymeric solutions and low viscosity complex fluids (e.g. inks, coating fluids, DNA solutions, blood). This section deals with the simulation using TransAT of a similar micro-rheometric device, consisting of Newtonian fluid flow through it, featuring a sudden planar contraction-expansion.

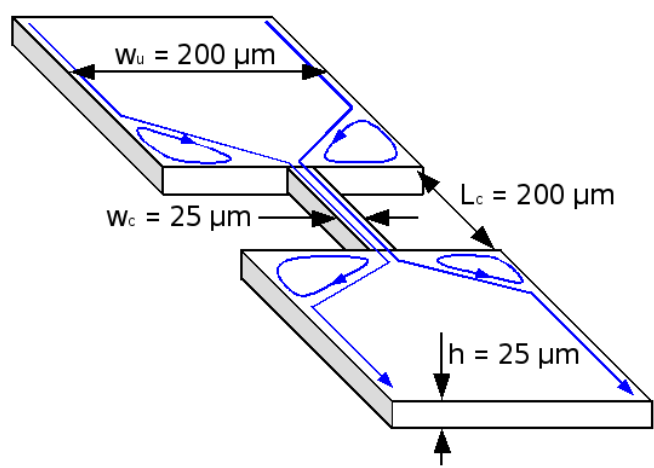

Fig. 3. Sketch and size of the microheometric device
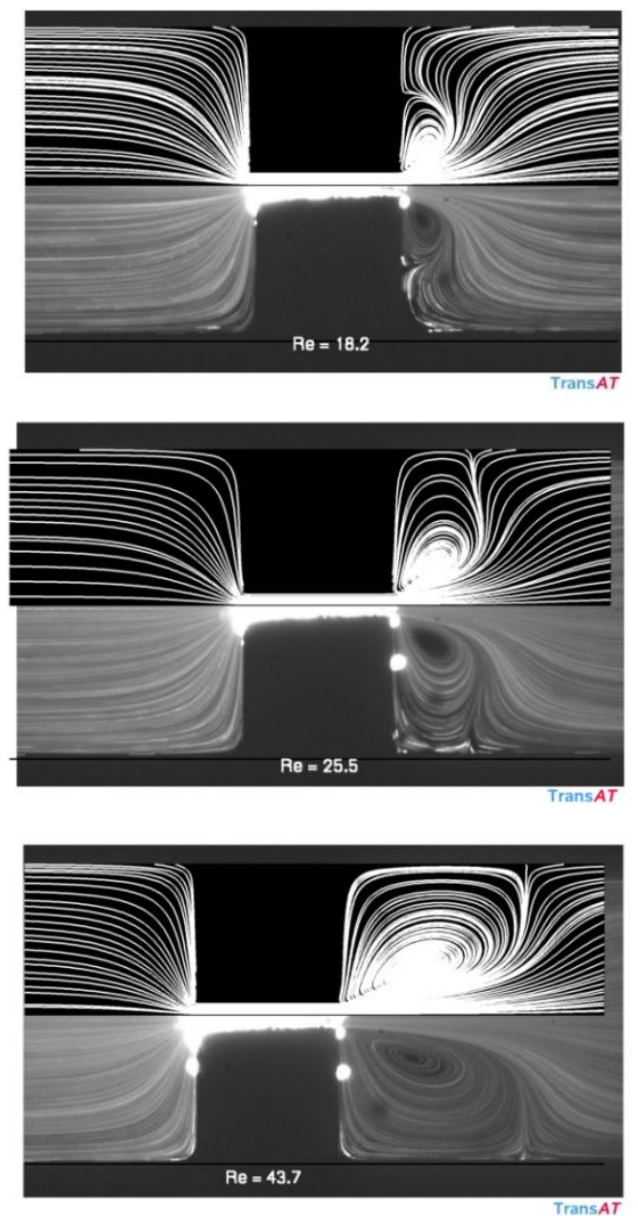

Fig. 4. Flow streamlines: TransAT CFD (upper) vs. measurements (lower) for $\mathrm{Re}=18.2-43.7$ 
The steady flow kinematics was characterized in the experiment of Oliveira et al. (2008) using fluorescent streak imaging and micro-particle image velocimetry (IPIV). A sketch and dimensions of the micro-geometry used in this investigation is presented in Fig. 4. The set of channels was designed to have approximately a 16:1:16 contraction-expansion ratio and different contraction lengths: $\mathrm{Lc}=100,200$ and $400 \mu \mathrm{m}$. In all cases, the channel depth, $\mathrm{h}=55 \mu \mathrm{m}$, the width of the upstream and downstream channels, wu $=\mathrm{wd}=400 \mu \mathrm{m}$, and the contraction width were kept constant. The mask used to produce the channels resulted in a final contraction width of wc $=26 \mathrm{~m}$ instead of the targeted $25 \mu \mathrm{m}$ required for the 16:1:16 contraction-expansion ratio; so in effect we have a 15.4:1:15.4 ratio. The length of the upstream and downstream channels is long enough that the flow reaches a fully developed profile at all flow rates.

The excess pressure-drop in the converging and diverging flow is an important dynamic measure to quantify if the device is intended to be used as a micro-fluidic extensional rheometer. To explore this idea, we examine the effect of the contraction length, aspect ratio and Reynolds number on the flow kinematics and resulting pressure field. Analysis of the computed velocity and pressure fields show that, for typical experimental conditions used in micro-fluidic devices, the steady flow is highly three-dimensional with open spiraling vortical structures in the stagnant corner regions. The flow is solved under laminar flow conditions, with the Navier Stokes equations kept as used for macro-flow simulations, i.e. without specific changes to the near-wall treatment to mimic the slip length. The simulations of the local kinematics and global pressure drop are in good agreement with experimental results as depicted in Fig. 5, comparing the flow streamlines along the device for variable flow Reynolds number. The device aspect ratio is shown to have a strong impact on the flow and consequently on the excess pressure drop. These simulations prove that for this size of devices, there is no need to account for microflow properties like adding slip length in the determination of wall shear.

\subsection{Flow in a Confined Jet Reactor}

We present here recent predictions of the flow field in a confined jet reactor for non-reactive flow conditions, compared to micro-PIV measurements of Gavi (2009). The CIJR micro-reactors are used in pharmaceutical industry to generate nano-scale powder. Here the system of equations (1-2) are solved directly without additional model, and without the inter-phase terms in the RHS of (2). The flow has been simulated under fixed and variable inflow conditions, on which flow unsteadiness actually depends. Figure 5 represents the geometry of the CIJR under consideration. The flow comes from two opposite pipes in the upper part of the reactor where the jets collide forming complex structures and then exit in the lower part. The flow Reynolds number in the reactor is based on its diameter and inflow velocity. Computations were carried out with the code TransAT. Solid boundaries of the reactor are represented with the IST explained above (c.f. Fig. 6). Since the walls are immersed in a Cartesian grid, meshing time is considerably reduced. With IST, the higher accuracy of the numerical scheme can be preserved since grid-skewness induced diffusion is eliminated. Fluid properties were selected by reference to the experiments: an aqueous solution of urea, with $\rho=1.141 \mathrm{~g} / \mathrm{cm}^{3}$ and $\mu=1.914 \mathrm{cPs}$.

Table 1 Nominal flow rates, mean velocities, residence times, Re \& Kolmogorov length scale

\begin{tabular}{|c|c|c|c|c|}
\hline $\begin{array}{c}\text { FR, } \\
\mathrm{ml} / \mathrm{min}\end{array}$ & $\begin{array}{c}\text { inlet velocity, } \\
\mathrm{m} / \mathrm{s}\end{array}$ & $\tau, \mathrm{s}$ & $\operatorname{Re}$ & $\begin{array}{c}\eta \eta, \\
\mu \mathrm{m}\end{array}$ \\
\hline 10 & 0.106 & 1.04 & 63 & 45 \\
\hline 20 & 0.212 & 0.52 & 126 & 27 \\
\hline 40 & 0.424 & 0.26 & 253 & 16 \\
\hline 90 & 0.955 & 0.115 & 569 & 9 \\
\hline
\end{tabular}

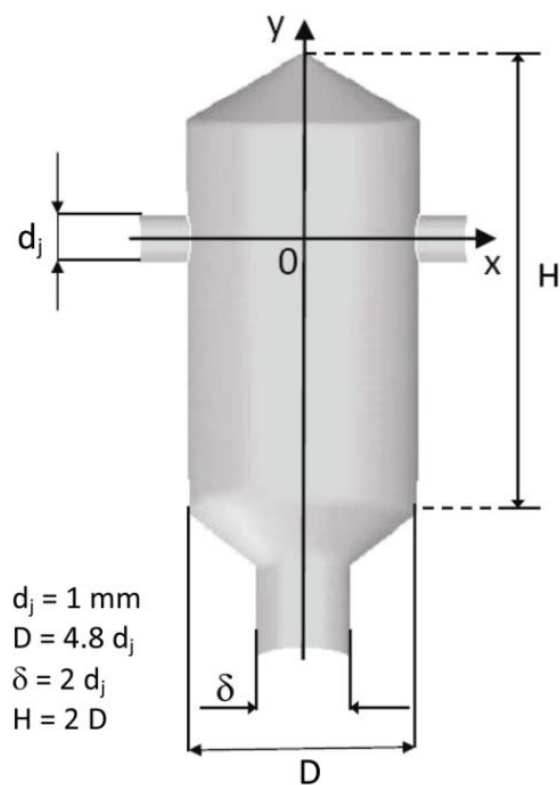

Fig. 5. Schematic geometry of the CIJR studied.

Four different inlet flow rates (FR) are imposed: 10, 20, 40 \& $90 \mathrm{ml} / \mathrm{min}$. The Reynolds numbers calculated using the inlet diameters and the mean velocities show that the flow regime in the pipe is laminar, thus parabolic velocity profiles have been imposed at the inlet. The first simulations were performed with inflow velocities calculated theoretically from the nominal flow rates imposed by the pumps (See Table 1). First, constant inflow conditions were applied, which show no flow perturbations as reported in the experiment. In a second phase, oscillating inflows were imposed, based on the experimental data, using a single harmonic oscillation in both the inflows. They were set to be in phase opposition in order to emphasize the effects of unsteady asymmetric flows. More details concerning the simulations are available in Icardi et al. (2009).

The oscillation amplitude was then set proportionally to the parabolic profile (to avoid negative inflow velocity) with a factor of 0.1 that fits the standard deviation obtained in the experiment with $\mathrm{FR}=10 \mathrm{ml} / \mathrm{min}$, which is the case where the external instabilities is more evident. Simulations were analyzed after the transient effects, when the influence of the initial condition 
disappears, between $\mathrm{t}=4 \tau$ and $8 \tau$. Figure 7 shows instantaneous vorticity magnitude fields with $\mathrm{FR}=90$ $\mathrm{ml} / \mathrm{min}$ and different inflow conditions. The left panel shows a quasi-steady behavior with large fluctuations. The neighboring panel depicts instead drastic changes in the flow behavior, featuring the development of more scales which are not created with constant inflows. The right variable inflows simulation reveal in right panel the existence of smaller scales generated from the breakup of the larger ones, responsible for the dissipation mechanism of turbulence. It is this process of scale generation which is important for phenomena like mixing, entrainment, and scalar diffusion.

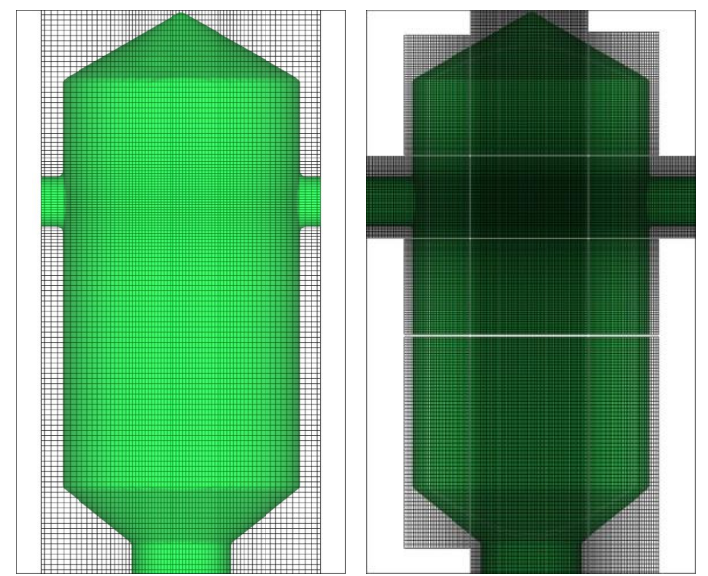

Fig. 6. The coarse single-block grid (n.1) used for simulations and the finest multi-block one (n.4).

Comparisons with experiments of Gavi (2009) are performed by analyzing the statistics of the flow along the inlet and outlet axes in the context of Fig. 8 (for the lowest FR value only). The comparison concerns the mean and root-mean-square (RMS) axial velocity profiles normalized by the maximum velocity, obtained with constant and unsteady oscillating inflows. When using constant inflows the simulation reaches a steady state in a short time. Although the mean velocity profile is close to the experiments the fluctuations are totally absent. With unsteady inflows instead, the model is better for both the mean and the fluctuations.
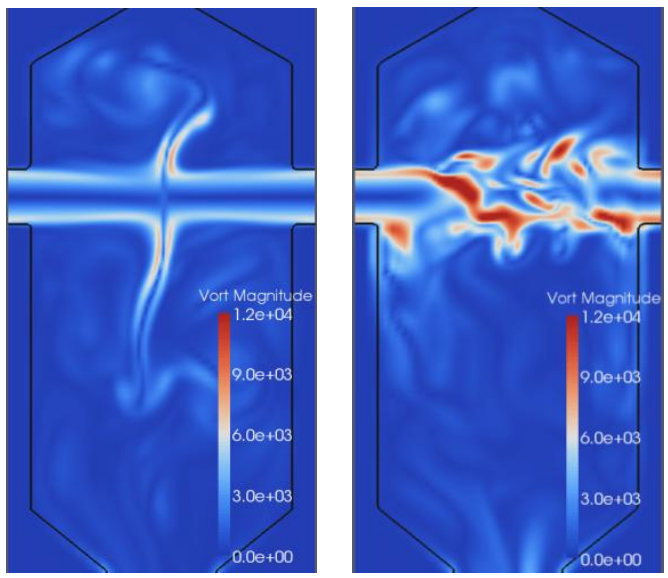

Fig. 7. Instantaneous vorticity magnitudes for constant (left) and unsteady inflows (right).
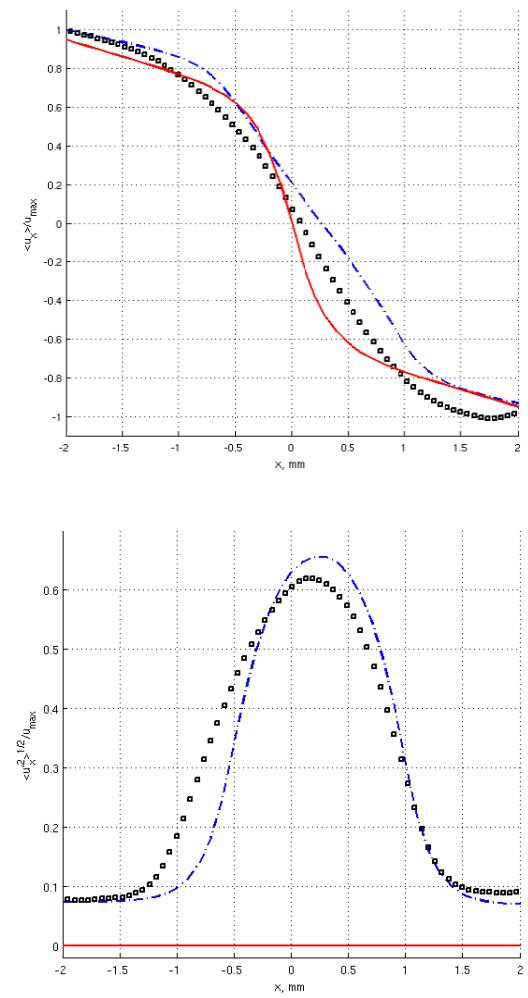

Fig. 8. Mean axial velocity along the $x$-axis for $F R=10$ $\mathrm{ml} / \mathrm{min}$. Comparison between constant (red) and unsteady inflows (blue) and mPIV (symbols).

\section{Multi-Phase Case Studies}

\subsection{Flow \& Heat Transfer in Heat Exchangers}

This example deals with the coupled two-phase fluid flow and conjugate heat transfer. Although it seems rather canonical, the test case is of importance in treating thermal management in heat exchangers for automatic industry in particular, and in large heat pumps employed for indoor air-conditioning. Shortly, a single pipe or a group of pipes of finite wall thickness is/are placed in a free turbulent stream, representing coolant air. Inside the pipe, hot water flows by the action of gravity or external pumping. The exercise consists in comparing the two extreme situations of the pipe being either completely filled or partially only. The heat transfer will be triggered by the coolant air to the core flow, through a triple-cycle mechanism: convective in the water, conductive through the pipe solid wall, and finally convective from the pipe surface to the outer core flow.

The IST approach is used to grid the set-ups (single pipe and group of pipes), similar to what is shown in Fig. 1 for the flow past a circular cylinder. A Cartesian mesh containing $98 \times 20 \times 66$ cells was employed for the single pipe and a finer one consisting of $138 \mathrm{x} 20 \mathrm{x}$ 130 cells for the group of five pipes. Five cells were used to cover the wall pipe. The Quick scheme was employed for all equations as discussed above. The level set was applied to track the water surface inside the pipes (when it was partially filled; where an inflow void fraction of $50 \%$ was set). 


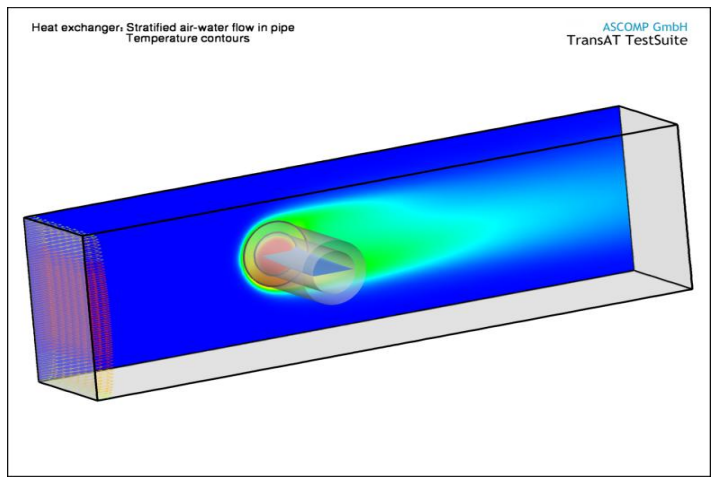

Fig. 9. 3D representation of the free surface in the pipe and heat transfer iso-contours.

The flow was solved in steady state conditions using the k- $\varepsilon$ model for turbulence combined with wall functions for the coolant air only (assuming a 5\% inflow turbulence intensity); the water flowing inside the pipe being laminar. The air Reynolds number based on pipe external radius is $\operatorname{Re}=7000$. The simulation result of the partially filled single-pipe is depicted in Fig. 9 above. It shows the inflow coolant, the free surface occupying the pipe, and the heat contours convected out by the coolant air. This result is of early steady state simulations performed to check the feasibility of such a coupled approach; surface deformations should be solved in transient, as for the other examples shown here. Figure 10 shows 2D sections of the pipe (taken at the centre) displaying the iso-contours of temperature in the completely and partially filled pipes.

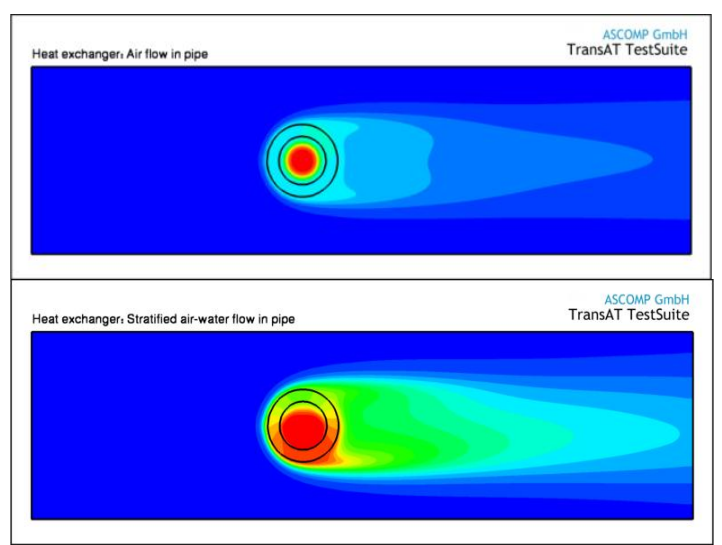

Fig. 10. Temperature contours for a completely filled pipe and a partially filled one.

The upper panel shows that the heat transfer from the pipe forced by the coolant externally is symmetrical around the pipe, as was to be expected. Indeed, a full unsteady simulation would show some loss of symmetry, if the flow Reynolds number is such that it triggers vortex shedding. The loss of heat transfer symmetry is depicted in the lower panel, where the heat is mostly directed towards the bottom occupied by the liquid, which has a higher conductivity. Note how the conductive heat transfer across the pipe wall is well coupled with the convection.

\subsection{TopSpot Droplet Generator}

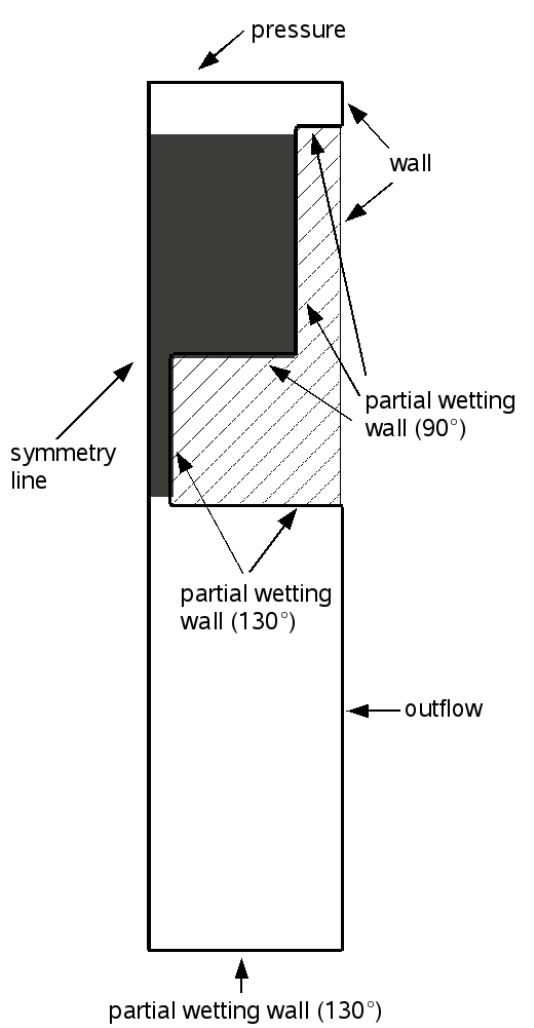

Fig. 11. Test case TopSpot- Domain and boundary conditions.

TopSpot is a microarray technology dedicated to generating hundreds of nanolitre droplets at once using an external pressure pulse. The simulation is performed as a validation case for the Level-Set implementation in TransAT, and in particular the triple-line dynamics model. The present comparisons based on the paper of Glatzel et al. (2008), in which the results of various other CFD softwares (Fluent, CFX, Flow-3D, and CFD-ACE+) were compared to experimental data. In contrast to the set-up in the mentioned paper, the current work is two-dimensional using the rotational symmetry, as can be seen in Fig. 11.

The overall domain size is $200 \times 880 \mu \mathrm{m}$ and contains 19 '564 cells, refined to the nozzle, whose diameter of $50 \mu \mathrm{m}$ is resolved by 20 cells. Therefore, the minimum grid spacing is $h=2.5 \mu \mathrm{m}$. The two fluids are air and water. The walls exposed to the gas-liquid interface are partially wetting, with a static contact angle of $130^{\circ}$. For driving the droplet generation a time dependent boundary condition was applied at the top of the domain. In this study, two different input pressure curves are compared: sinusoidal and harmonic signals. The points mark significant data of the experimentally measured pressure curve. Reconstructed sinusoidal and harmonic pulses were used to closely reproduce the characteristic points of the experimental curves, and used separately for simulation. 


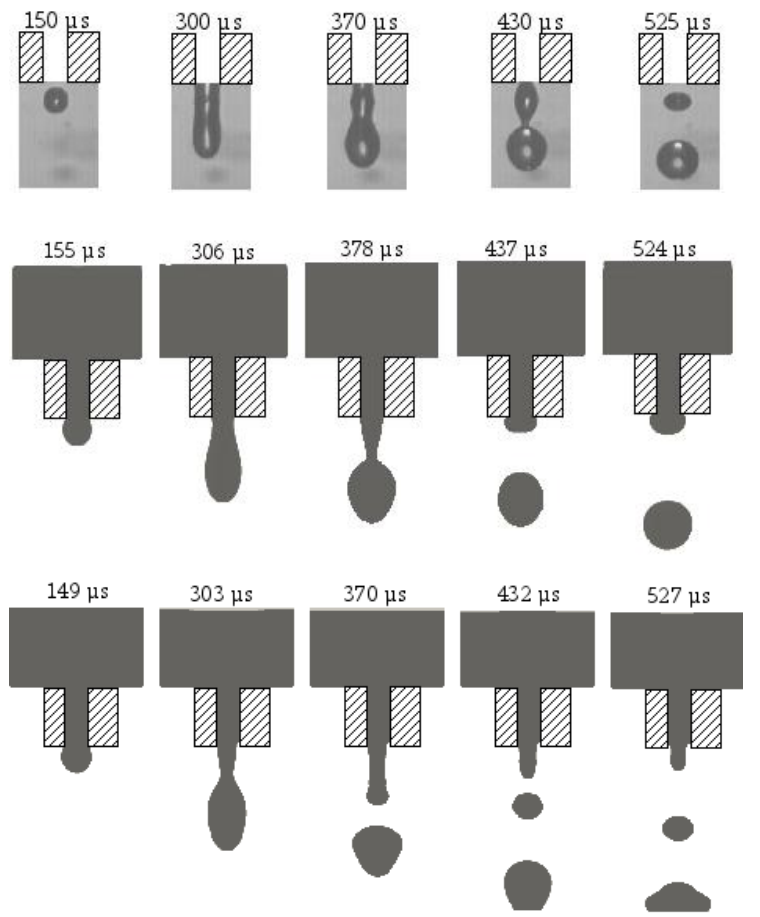

Fig. 12. Comparison between experiments and TransAT (sinus pulse; 1st panel, and harmonic pulse; 2nd panel).

The detailed CMFD analysis of the flow can be found in Miehe and Narayanan (2009). The adaptive time stepping in TransAT is based on several different non-dimensional numbers to control the change in the time step, viz. numbers accounting for convection (CFL), diffusion, surface tension, gravity, etc. Adaptive time stepping is crucial to stably resolve phenomena such as droplet pinch-off. The time step through the course of the simulation varied between 10ns and $100 \mathrm{~ns}$.

This fully transient problem is solved by TransAT within 1 hour on a single CPU. The results using the two pressure boundary conditions compared with the experiments according to Glatzel et al. (2008) are presented in Fig. 12. The sine pressure curve is not sufficient to produce the same droplet characteristics as given in the experimental data. The tear-off time of $339 \mu$ s is too early compared with the experimental value of $450 \mu \mathrm{s}$. In addition, a satellite drop is produced enlarging the volume of the dispersed fluid shown for $432 \mu \mathrm{s}$ in Fig. 12b. Figure 12c presents the results for the harmonic pressure pulse. The tear-off time of $394 \mu$ s is also better, compared to the former results. The droplet volume of $0.9 \mathrm{nl}$ is larger than the experimental value of $0.68 \mathrm{nl}$, however, the value is in the range of the two successfully tested CFD codes in the above mentioned paper (Glatzel et al. 2008) (0.66 nl for CFD-ACE+ and $1.4 \mathrm{nl}$ for CFX). We note that compared to all other codes only Trans $A T$ predicts a teared-off droplet remaining attached to the nozzle.

\section{CONCLUSIONS}

The paper reports the progress made in predicting a variety of complex, multi-scale, multi-physics flows using the CMFD code TransAT. By purpose we have omitted to report turbulent flow results to focus on an ever-growing class of flows that are actually more demanding than conventional CFD. Our contribution addresses in particular the predictive capability of the code in treating microscale flows and interfacial multiphase flows with triple-lines dynamics, combined with heat transfer, using modern Interface Tracking Techniques like level sets. Complex 2D and 3D examples shown were treated using a new version of the code that uses the Immersed Surfaces Technique to map complex components into simple rectangular Cartesian grids. It is shown that complex multi-scale, multi-physics flow problems are today within reach of modern CMFD codes using tailored computational techniques, with relatively fast response times.

\section{REFERENCES}

Gavi, E., D.L. Marchisio and A. Barresi (2006).CFD modeling and scale-up of Confined Impinging Jet Reactors. Chem. Eng. Sci. 62, 2228-224.

Glatzel, T. et al. (2008). Computational fluid dynamics (CFD) software tools for microfluidic Applications - A case study. Computers and Fluids 37, 218-235.

Hirt, C.W. and B.D. Nichols (1981). Volume of fluid (VOF) method for the dynamics of free boundaries. J. Comput. Phys. 39, 205-226.

Icardi, M., E. Gavi, D.L. Marchisio, M.G. Olsen, R.O. Fox, A.A. Barresi, and D. Lakehal (2011). Investigation of the flow field in an axial symmetric Confined Impinging Jets Reactor by means of mPIV and DNS. Chem. Eng. Journal, 166, 294-305.

Lakehal, D., M. Meier and M. Fulgosi (2002). Interface tracking for the prediction of interfacial dynamics and heat transfer in multiphase flows. Int. J. Heat \& Fluid Flow 23, 242-257.

Mittal, R. and G. Iaccarino (2005). Immersed Boundary Methods. Ann. Rev. Fluid Mech 37, 239-261.

Miehe, A. and C. Narayanan (2009, May). Prediction of droplet tear-off and meniscus formation in the top-spot experiment using TransAT. In Proc. NSTI/Nanotech Conf., Huston Texas, USA.

Oliveira, M.S.N., L.E. Rodd, G.H. McKinley, and M.A. Alves (2008). Simulations of extensional flow in microrheometric devices. J. Microfluidics \& Nanofluidics 5, 6, 809-826.

Sussman, S., P. Smereka and S. Osher (1994). A Level set Approach for computing incompressible two-phase flow. J. Comp. Physics 114, 146-161.

TransAT (2011, Accessed). ASCOMP GmbH software package TransAT (Transport phenomena Analysis Tool). www.ascomp.ch/transat. 